\title{
Comparison of midline and off-midline lingual vibrotactile threshold responses in men and women
}

\author{
DONALD FUCCI, JIM CANTRELL, LINDA PETROSINO, and RANDALL R. ROBEY \\ School of Hearing and Speech Sciences, Ohio University, Athens, Ohio 45701
}

\begin{abstract}
Lingual vibrotactile thresholds were obtained from 10 young adult men and 10 young adult women on three different lingual test sites at a frequency of $250 \mathrm{~Hz}$. The lingual test sites chosen for study were: (1) the dorsal midline of the anterior portion of the tongue, (2) a point halfway between the dorsal midline and the right upper lateral border of the tongue, and (3) a point halfway between the dorsal midline and the left upper lateral border of the tongue. Results showed that thresholds obtained on the dorsal midline of the tongue were not statistically significantly different for the men and women, but thresholds obtained off midline in either direction were statistically significantly different for the two groups of subjects. Compared with the midline thresholds, the off-midline thresholds were seen to become higher for the male subjects, but they were seen to become lower for the female subjects. Implications of these results for future testing are discussed, and further research dealing with off-midline lingual vibrotactile measurement is recommended.
\end{abstract}

The controlled study of lingual vibrotactile threshold sensitivity has been an ongoing process that had its beginnings in the early 1950s and has received a great deal of attention in the 1960s and 1970s (Fucci, 1972; Sherrick, 1953; Verrillo, 1966). All studies to date have concentrated on obtaining vibrotactile thresholds from the dorsal midline of the tongue at some variable distance from the tongue tip. This site is routinely chosen as a matter of convenience, in that it is readily accessible and definable (Fucci \& Crary, 1979).

A review of the literature concerned with vibratory thresholds of various body parts warrants study of lingual areas other than dorsal midline in that it provides information that indicates that various regions of the body will definitely yield different threshold responses, even though they are part of the same structure. For example, a number of investigators have looked at vibrotactile threshold comparisons between upper and lower as well as right and left halves of the body. There is a general agreement that the upper extremities are more sensitive than the lower extremities (Marsh, 1980; Weinstein, 1968), but there is some confusion in terms of thresholds related to right and left sides of the body. Cosh (1953) and Steiness (1957) studied a large number of subjects by obtaining vibratory thresholds from the right and left index fingers, and they found no differences. Weinstein (1968) and Whanger and Wang (1974) performed similar studies and found the nondominant side of the body to be more sensitive to vibration than the dominant side. Cosh (1953) also focused on different parts of the hand and found the fingertip to be far more sensitive to vibration than the fleshy pad over the first metacarpal.

Even though vibrotactile thresholds have not been obtained from different regions of the tongue, there are reports suggesting that the oral cavity taken as a whole does not demonstrate uniformity in its mode of response to stimulation (Ringel, 1970). Two-point discrimination studies performed by Ringel and Ewanowski (1965) have definitely shown that the front of the mouth is more sensitive than its posterior regions, and increased discrimination ability exists at the midline of the structure when compared with off-midline sites.

The literature, for the most part, does not suggest differences between the vibrotactile thresholds for men and those for women for the various body parts tested (Plumb \& Meigs, 1961; Steinberg \& Graber, 1963; Verrillo, 1979; Verrillo \& Ecker, 1977). There are, however, a few exceptions. Steiness (1957) studied 100 normal adults using a bioesthesiometer and found women's thresholds to be better than those of men for the lower body extremities. Goff, Rosner, Detre, and Kennard (1965) studied 417 normal subjects and found men to have lower vibratory thresholds than women for the left index finger.

The present pilot work was designed to begin the study of off-midline lingual vibrotactile threshold responses in comparison with midline lingual vibrotactile threshold responses for normal young adult men and women.

\section{METHOD}

\section{Subjects}

Twenty individuals (10 male and 10 female) were randomly selected from students and employees of Ohio University to form the two experimental groups required for this study. The 10 male subjects ranged in age from 18 to 30 years, with a mean age of 24 years, and the 10 female subjects ranged in age from 
18 to 27 years, with a mean age of 20 years. None of the subjects reported present or past histories of speech or hearing disorders or any known sensory and/or motor impairments.

\begin{abstract}
Apparatus
The vibrotactile instrumentation used in this study consisted of a stimulus unit and a measurement unit. The stimulus unit was composed of a sine-wave generator, an electronic switchinterval timer, an amplifier, a 2-dB-step variable attenuator, and a vibrator with a probe-contactor extension. The pulsed vibratory signal generated had a $50 \%$ duty cycle (on $.5 \mathrm{sec}$ and off $.5 \mathrm{sec}$ ), with a rise and decay time of $100 \mathrm{msec}$. The measurement unit included an accelerometer, a cathode follower, a microphone amplifier, and a voltmeter. A narrow-band noise generator was used to present auditory masking at $70 \mathrm{~dB}$ HTL to all subjects through TDH-39 headphones. A more detailed description of the vibrotactile equipment and procedures can be found in a comprehensive review of the state of the art by Fucci and Crary (1979).
\end{abstract}

\section{Procedure}

Each subject was seated in an adjustable chair and asked to extend his tongue between two sterilized plastic disks. The lower clamping disk was then raised to a comfortable height for each subject. The clamping apparatus acted as a lingual support for the subject's comfort and was not utilized to compress the tongue in any way. A hole in the top disk provided access for the vibrator probe to the anterior midline section of the dorsum of the tongue. The $.128-\mathrm{cm}^{2}$ contactor on the end of the probe was lowered until a voltmeter recorded contact with the tongue. It was then lowered $1 \mathrm{~mm}$ farther into the lingual surface in order to assure constant displacement of the contactor on the tongue for all subjects.

Prior to testing, all subjects received training to acquaint them with the testing apparatus. Subsequent to adequate training, standardized instructions were read to each subject during the testing session. The three different lingual testing sites were designated as dorsal midline of the anterior portion of the tongue, a point halfway between the dorsal midline and the right upper lateral border of the tongue, and a point halfway between the dorsal midline and the left upper lateral border of the tongue. Testing was randomized for order of threshold determination on the three different lingual testing sites. Three thresholds were obtained for each of the three test sites, for a total of nine threshold measurements per subject. The single test frequency used was $250 \mathrm{~Hz}$, and each subject was required to respond by raising his hand as soon as the pulsed vibratory signal was detected. An ascending psychophysical method of limits was employed throughout the experiment. The mean of the three millivolt readings obtained from each test site was accepted as lingual threshold for that site and was later converted to displacement in microns with the standard " $\mathrm{g}$ " formula for acceleration conversion.

\section{RESULTS AND DISCUSSION}

The threshold means and standard deviations for the male and female subjects obtained from the three lingual test sites are shown in Table 1. The thresholds for the men appear to be different from those for the women in that the women show lower thresholds for all three sites being tested. The threshold differences between groups appear to be far more exaggerated with respect to the off-midline conditions. The standard deviations for both groups are small for all experimental conditions, indicating reliable measurement throughout.

The data were subjected to a multivariate analysis of variance for a two-group repeated-measures design
Table 1

Mean Sensory Threshold Values (in Microns) and Standard Deviations for the Two Groups Across the Three Experimental Conditions

\begin{tabular}{lcccccccc}
\hline & \multicolumn{2}{c}{ Left } & & \multicolumn{2}{c}{ Midline } & & \multicolumn{2}{c}{ Right } \\
\cline { 2 - 3 } & Mean & SD & & Mean & SD & & Mean & SD \\
\hline Male & .895 & .367 & & .736 & .351 & & .955 & .414 \\
Female & .526 & .286 & .642 & .316 & .543 & .245 \\
\hline
\end{tabular}

(Stevens \& Barcikowski, in press). The overall analysis, which is summarized in Table 2 , indicated no statistically significant differences between subject groups or test sites by themselves, but it did show statistical significance for a Group by Test Site interaction at the .05 alpha level. Post hoc contrasts were conducted at the .05 alpha level in order to more accurately define the nature of the interaction effect. The contrasts revealed statistically significant differences between the men's and women's thresholds for both of the off-midline lingual test sites, but not for the dorsal midline test site.

The statistical analyses suggest that vibrotactile thresholds obtained from the dorsal midline of the tongue are similar for both male and female subjects. Vibrotactile thresholds obtained from the off-midline lingual areas, however, do show differences between the sexes. The off-midline thresholds, when compared with the midline thresholds, appear to become higher for the male group and lower for the female group. This trend seems to exist for both right and left sides of the tongue.

The threshold results obtained from the dorsal midline are compatible with those studies that indicate that thresholds of vibration taken from various body parts do not show significant differences between men and women (Plumb \& Meigs, 1961; Steinberg \& Graber, 1963; Verrillo, 1979; Verrillo \& Ecker, 1977). Those threshold results obtained from the off-midline lingual sites are more compatible with the findings of Steiness (1957), who found differences between the sexes for the lower body extremities, and Goff et al. (1965), who found men to have vibratory thresholds different from those of women for the left index finger.

At this point in time, there is no clear explanation why the off-midline threshold testing conditions led to differences in thresholds between the sexes. There is also no substantive information that would lead to the expectation that the males would have significantly higher thresholds than the females for the off-midline

Table 2

Summary for Analysis of Variance Performed to Determine if Vibrotactile Thresholds Differed As a Function of Sex and Lingual Test Site

\begin{tabular}{lccc}
\hline & Wilks' Lambda & df & Probability \\
\hline Sex & .804 & $1,1,18$ & $>.05$ \\
Site & .935 & $2,1,18$ & $>.05$ \\
Sex by Site & .504 & $2,1,18$ & $<.05$ \\
\hline
\end{tabular}


lingual regions, irrespective of the side of the tongue being studied.

The nature of these findings would lead to the reasonable suggestion that future examiners measuring lingual vibrotactile thresholds exercise caution in selecting subjects and/or site of measurement. If the examiner is concerned with minimizing within-group error, the sample should be homogeneous with respect to sex. If homogeneity with respect to sex is not feasible or desirable, then it is important that thresholds be taken on the midline of the tongue, where differences between the sexes appear to be minimized or nonexistent.

A clearer understanding of off-midline lingual vibrotactile threshold characteristics for both men and women is necessary. To our knowledge, this is the first study to deal with the off-midline testing mode, and the need for more normative data for men and women of all ages is obvious.

\section{REFERENCES}

Cosh, J. A. Studies on the nature of vibration sense. Clinical Science, 1953, 12, 131-150.

Fucci, D. Oral vibrotactile sensation: An evaluation of normal and defective speakers. Journal of Speech and Hearing Research, 1972, 15, 179-184.

Fucci, D., \& Crary, M. A. Oral vibrotactile sensation and perception: State of the art. In N. Lass (Ed.), Speech and language: Advances in basic research and practice. New York: Academic Press, 1979.

Goff, G. D., Rosner, B. S., Detre, T., \& Kennard, D. Vibration perception in normal men and medical patients. Journal of Neurology and Psychiatry, 1965, 28, 503-509.

Marsh, G. R. Perceptual changes with aging. In E. W. Busse \& D. G. Blazer (Eds.), Handbook of geriatric psychiatry. New York: Van Nostrand Reinhold, 1980.
Plumb, C. S., \& Meigs, J. W. Human vibration perception: Part 1. Vibration perception at different ages. Archives of General Psychiatry, 1961, 4, 611-614.

RINGEL, R. L. Oral sensation and perception: A selective review. In R. T. Wertz (Ed.), Speech and the dentofacial complex: The state of the art. Washington, D.C: American Speech and Hearing Association, 1970.

Ringel, R. L., \& EWANowski, S. J. Oral perception: 1. Two-point discrimination. Journal of Speech and Hearing Research, 1965, 8, 389-398.

SHERrick, C. E. Variables affecting sensitivity of the human skin to mechanical vibration. Journal of Experimental Psychology, 1953, 45, 273-282.

Steinberg, F. U., \& Graber, A. L. The effect of age and peripheral circulation on the perception of vibration. Archives of Physical Medicine and Rehabilitation, 1963, 44, 645-650.

STE INESS, J. Vibratory perception in normal subjects. Acta Medica Scandinavica, 1957, 158, 315-325.

Stevens, J. P., \& Barcikowski, R. S. Applied multivariate statistics for the social sciences. Washington, D.C: University Press of America, in press.

Verrillo, R. T. Specificity of a cutaneous receptor. Perception \& Psychophysics, 1966, 1, 149-153.

Verrillo, R. T. Comparison of vibrotactile threshold and suprathreshold responses in men and women. Perception \& Psychophysics, 1979, 26, 20-24.

Verrillo, R. T., \& Ecker, A. D. Effects of root or nerve destruction on vibrotactile sensitivity in trigeminal neuralgia. Pain, 1977, 3, 239-255.

WEINSTEIN, S. Intensive and extensive aspects of tactile sensitivity as a function of body part, sex, and laterality. In D. R. Kenshalo (Ed.), The skin senses. Springfield, Ill: Thomas, 1968.

Whanger, A. D., \& Wang, H. A. Clinical correlates of the vibratory sense in elderly psychiatric patients. Journal of Gerontology, 1974, 29, 39-45.

(Received for publication December 23, 1981.) 